\title{
Caracterización agronómica de 95 accesiones en el banco de germoplasma de café en Chanchamayo (Perú), año 2016
}

\section{Agronomic characterization of 95 accessions in the bank of germplasm of coffe plant in Chanchamayo (Peru) year 2016}

\author{
L. Alvarado, R. Vértiz, J. Jiménez, R. Borjas, V. Castro y A. Julca \\ Universidad Nacional Agraria La Molina, Lima, Perú
}

Recibido el 14 de junio del 2017. Aceptado el 19 de julio del 2017

DOI: https://doi.org/10.33017/RevECIPeru2017.0004/

\section{Resumen}

El presente estudio tuvo como objetivo evaluar las características agronómicas de 95 accesiones en el banco de germoplasma de café (Coffea arabica L.) en Chanchamayo, año 2016. Este trabajo se llevó a cabo en el fundo "La Génova" de la Universidad Nacional Agraria La Molina en San Ramón-Chanchamayo. La caracterización se basó en caracteres que describan la capacidad productiva de la planta y su comportamiento frente a la "broca" (Hypothenemus hampei) y "roya" (Hemileia vastatrix). Luego se realizó un análisis de varianza y una Prueba de Duncan para cada variable estudiada mediante el programa Statgraphic. Se realizó un análisis multivariado (componentes principales y agrupamientos) con el programa NTSYS que permitió conocer los caracteres que han expresado un mayor grado de variabilidad, así como la relación entre accesiones. Las accesiones con más altos rendimientos en promedio de café cerezo/planta fueron UNACAF70 con $5.42 \mathrm{~kg} /$ planta y UNACAF-149 con $4.16 \mathrm{~kg} / \mathrm{planta}$; mientras que las accesiones menos sobresalientes fueron UNACAF-151 con $0.22 \mathrm{~kg} /$ planta y UNACAF-223 con $0.15 \mathrm{~kg} /$ planta. Con respecto al peso de café pergamino seco por planta, la accesión más sobresaliente siguió siendo UNACAF-70 con $1.1 \mathrm{~kg} / \mathrm{planta}$. Finalmente, los caracteres más discriminantes fueron peso de café cerezo, peso de café pergamino seco, número de frutos por planta, peso de pulpa de 100 frutos maduros y peso de 100 frutos maduros.

Descriptores: café, broca del café, roya del café, análisis multivariado.

\begin{abstract}
The present study aimed to evaluate the agronomic characteristics of 95 accessions in the coffee germplasm bank (Coffea arabica L.) in Chanchamayo, in 2016. This work was carried out in "La Genova" fund of the National Agrarian University La Molina in San Ramón-Chanchamayo. The characterization was carried out on the basis of characters that describe the productive capacity of the plant and its behavior against the "drill" (Hypothenemus hampei) and "rust" (Hemileia vastatrix). An analysis of variance and a Duncan test were performed for each variable studied using the Statgraphic program. Then a multivariate analysis (main components and groupings) was carried out with the NTSYS program that allowed to know the characters that have expressed a greater degree of variability, as well as the relation between accessions. The accessions with the highest yields in average coffee cherry / plant were UNACAF-70 with $5.42 \mathrm{~kg} /$ plant and UNACAF149 with $4.16 \mathrm{~kg} /$ plant; While the least outstanding accessions were UNACAF-151 with $0.22 \mathrm{~kg} /$ plant and UNACAF-223 with $0.15 \mathrm{~kg} /$ plant. Regarding the weight of dry parchment coffee per plant, the most outstanding accession remained UNACAF-70 with $1.1 \mathrm{~kg} /$ plant. Finally, the most discriminating characters were cherry coffee weight, dry parchment coffee weight, number of fruits per plant, pulp weight of 100 mature fruits and weight of 100 mature fruits.
\end{abstract}


Key words: coffee plant, coffee berry borer, coffee rust disease, multivariate analysis.

\section{Introducción}

La producción del café (Coffea arabica L.) es crucial para una serie de países tropicales como Brasil, México, Colombia, Etiopía y Kenia [4]. Esta especie tiene su origen en las selvas tropicales de la parte sur-occidental de Etiopía y representa aproximadamente el $70 \%$ de la producción mundial, usándose para la preparación de bebidas de alta calidad [3], que son muy apreciadas por su aroma, sabor y efecto estimulante [15]. En el Perú, la caficultura tiene importancia económica, social y ambiental, debido a que se desarrolla en 11 regiones, contando con alrededor de $425 \mathrm{mil}$ hectáreas sembradas [9]. Además, se estima que unas 223 mil familias se dedican a la siembra de café y otras dos millones de personas están incluidas en la cadena de producción de este grano [12]. Históricamente, en nuestro país, la producción de este grano aromático, se ha basado en el uso de algunas pocas variedades como Típica y Caturra Roja y, en menor escala, en Pache, Mundo Novo, Bourbon, y más recientemente, Catimor. Para ampliar la gama de variedades comerciales que el caficultor peruano pueda usar; es necesario el conocimiento adecuado de éstas. Un primer paso fue la instalación de un banco de germoplasma, por ello, la Fundación Para el Desarrollo Agrario (FDA) de la Universidad Nacional Agraria La Molina (UNALM), con el apoyo financiero del FINCyT y en alianza con Café Perú, realizó el proyecto "Estudio de la variabilidad genética del café y establecimiento de un banco de germoplasma en la selva peruana" (2009 - 2011).

Después de la instalación del banco de germoplasma de café en el Fundo La Génova" de la UNALM en San Ramón (Chanchamayo), quedó la tarea de hacer la caracterización de los genotipos colectados; ya que, además de ser un proceso esencial para el manejo de los recursos fitogenéticos, permite conocer las características de interés para el mejoramiento genético [4]. Es por ello, que este trabajo tuvo como objetivo evaluar las características agronómicas de 95 accesiones en el banco de germoplasma de café (Coffea arabica L.) en Chanchamayo, año 2016.

\section{Materiales y métodos}

La fase experimental se realizó en el Banco de Germoplasma ubicado en el Fundo "La Génova" del Instituto Regional de Desarrollo de la Selva
(IRD-Selva) de la Universidad Nacional Agraria La Molina (UNALM); ubicado en el distrito de San Ramón, provincia de Chanchamayo, departamento de Junín, a una altitud de 965 m.s.n.m., 1105.790” de latitud sur y 75020.969' latitud oeste. En la zona, la temperatura mínima se registra en los meses de Junio $\left(21.47^{\circ} \mathrm{C}\right)$ y Julio $\left(21.32^{\circ} \mathrm{C}\right)$ y la máxima en Octubre $\left(23.76{ }^{\circ} \mathrm{C}\right)$. El mayor porcentaje de humedad relativa se presenta en Enero $(89.79 \%)$ y la mínima en el mes de Octubre $(76.52 \%)$. El material vegetal evaluado correspondería a las variedades: Catimor, Caturra, Caturra, Catuaí, Pache, Bourbon, Mundo Novo, Típica, Gran Colombia, Colombia, Geisha, Costa Rica 95, Maragogype y Villalobos; una colección de diversos departamentos del país [10]. Las plantas tuvieron 6 años de edad, siendo la campaña 2015-2016 su tercera y más importante cosecha obtenida (Figura 1 y 2 ).

La caracterización agronómica del Banco de Germoplasma se realizó evaluando las variables relacionadas a la capacidad productiva de las plantas: peso de café pergamino seco ( $\mathrm{g} /$ planta), peso de café pergamino seco (qq/ha), relación CC/CPS, peso de 100 semillas (g) y las relacionadas a su respuesta a plagas y enfermedades: el nivel de infestación de la broca (\%) y el nivel de incidencia de la roya (\%) (Tabla 1). Cada accesión cuenta con una hilera de 9 plantas, para la evaluación sólo se consideró las 5 plantas centrales; es decir, el número de tratamientos analizados fueron 95 (número de accesiones) con 5 repeticiones ( 1 planta $=1$ repetición). Los datos obtenidos de la caracterización agronómica fueron analizados con el programa Statgraphic. Mediante este software se obtuvo las medidas estadísticas (media, coeficiente de variación, varianza, valor mínimo y valor máximo) que permitieron tener una visión general de la variabilidad de las accesiones encontradas en el Banco de Germoplasma en estudio. Finalmente, se realizó un Análisis de Componentes Principales que permitió conocer los caracteres que expresan mayor variabilidad y un Análisis Multivariado de Agrupamiento para determinar la forma en que se relacionan las accesiones evaluadas.

Tabla 1: Variables Estudiadas en el Banco de Germoplasma

\begin{tabular}{|l|l|}
\hline & \multicolumn{1}{|c|}{ Variables Estudiadas } \\
\hline Capacidad & Peso de café pergamino seco (g/planta) \\
\cline { 2 - 2 } Productiva & Peso de café pergamino seco (qq/ha) \\
\hline
\end{tabular}




\begin{tabular}{|l|l|}
\hline \multirow{2}{*}{} & Peso de 100 semillas $(\mathrm{g})$ \\
\cline { 2 - 2 } & Relación CC/CPS \\
\hline $\begin{array}{l}\text { Incidencia de } \\
\text { plagas y } \\
\text { enfermedades }\end{array}$ & Incidencia de Roya (\%) \\
\cline { 2 - 2 } & Nivel de infestación de Broca (\%) \\
\hline
\end{tabular}



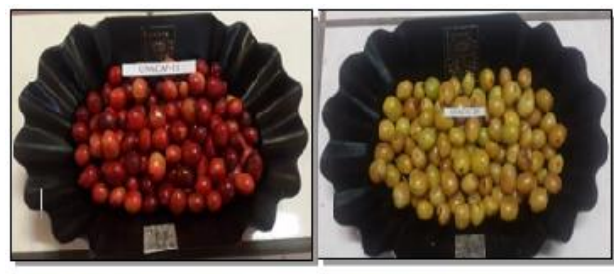

UNACAF-11 Var. G. Culumbia)

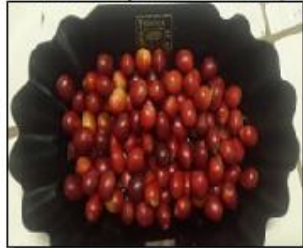

IVACAF-74 (Var. Cavimor)

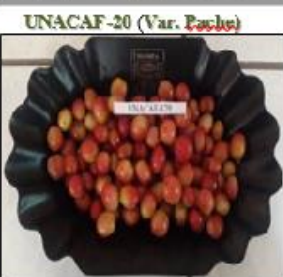

UNACAF-129 (Var. Borbön)
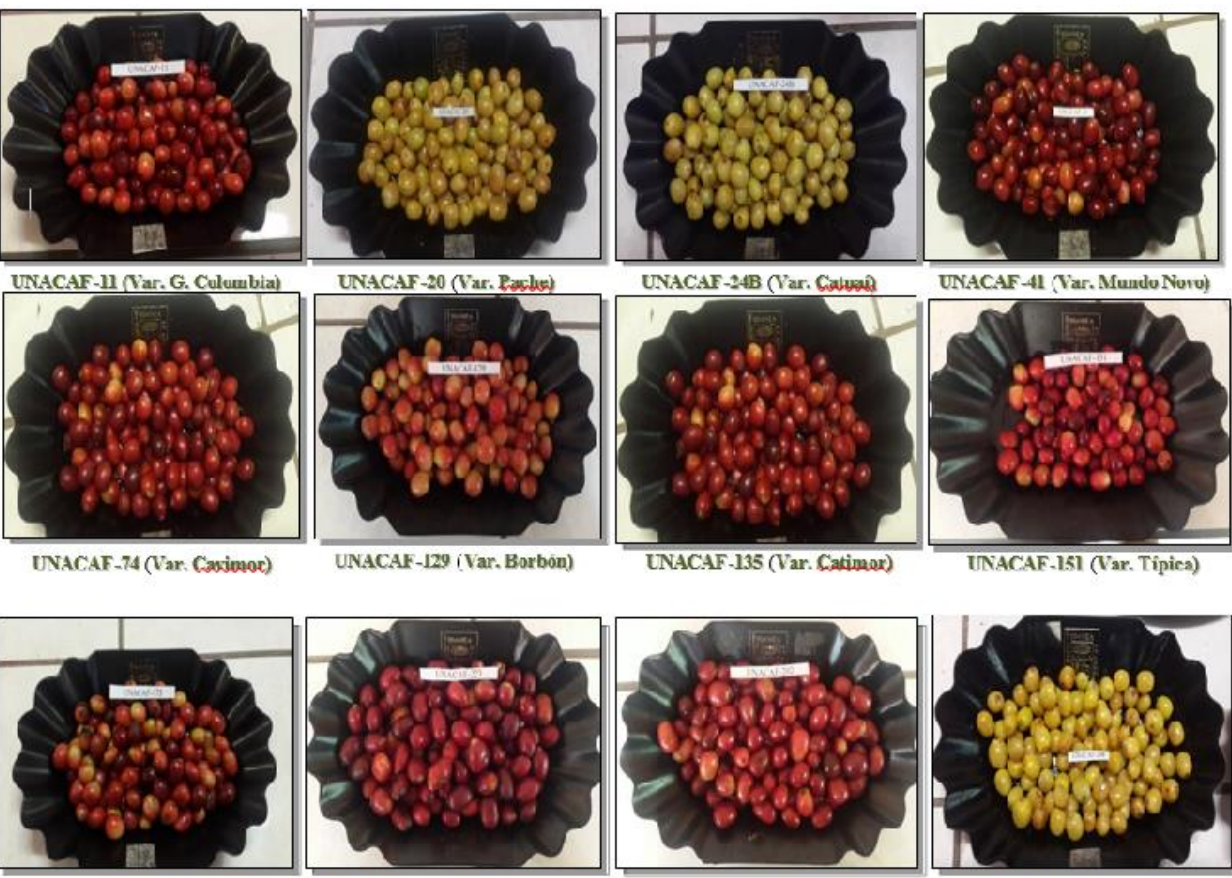

UNACAF-173 (Nar. CR 92)
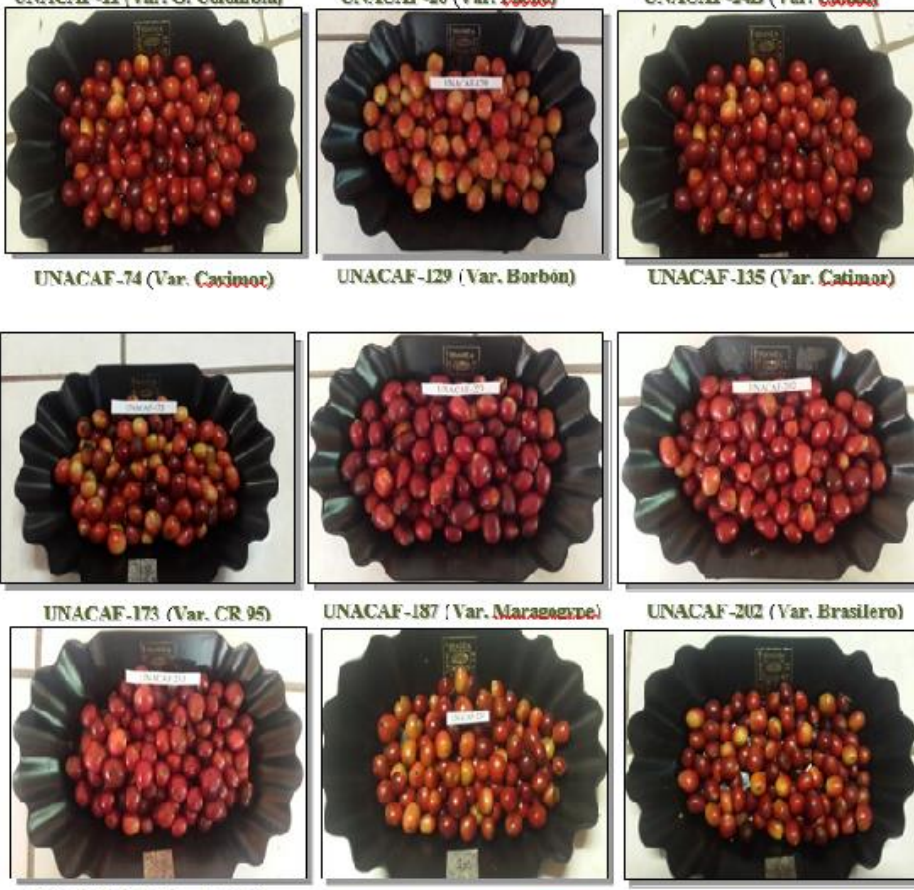

UNACAF-213 Var. Geisha)

UNACAT-220 (Var. Catimex)

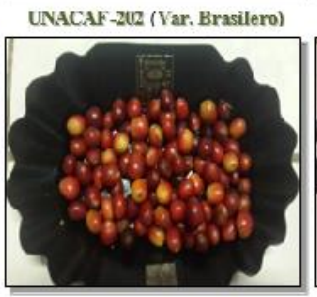

UNACAF 22 (Var. Villalobee)

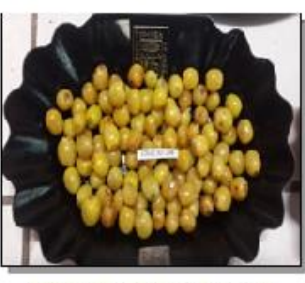

UNACAF-208 Nar. Caurra)

Figura 1: Café cerezo de accesiones evaluadas en el Banco de Germoplasma, Chanchamayo. Año 2016.
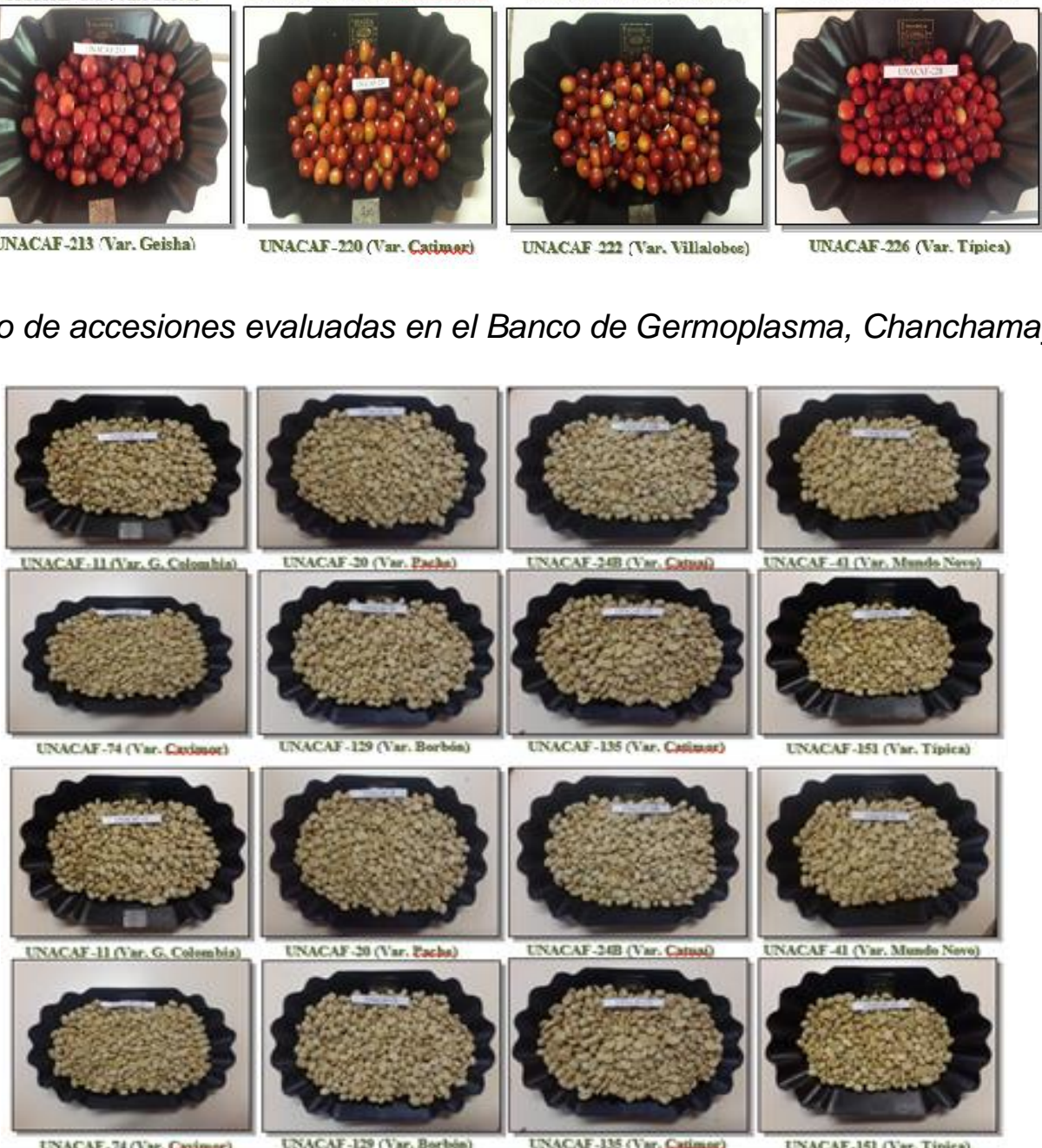

UNACAF-226 (Var. Típiea)
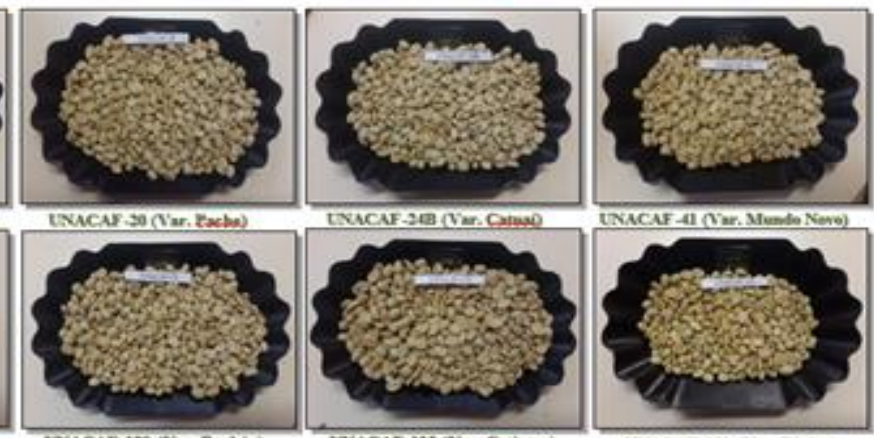

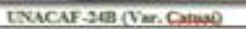
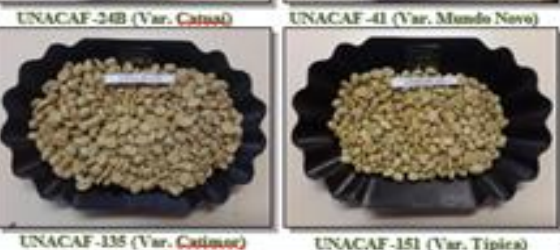

Figura 2: Café pergamino seco de accesiones evaluadas en el Banco de Germoplasma, Chanchamayo. Año 2016. 


\section{Resultados y discusiones}

\subsection{Peso de cosecha de café pergamino seco}

El peso de café pergamino seco es afectado principalmente por la calidad del café cerezo y por la tecnología utilizada para el beneficio (tipo y calibración) [13]. Esta variable es importante al momento de evaluar el rendimiento del cultivo debido a que en esta forma es comercializable por el agricultor. Los resultados del análisis de variancia indican que existieron diferencias significativas entre los tratamientos, el coeficiente de variabilidad fue de $36.57 \%$.

La prueba de Duncan a un nivel de 0.05 indica que la accesión con mayor peso de cosecha en café cerezo fue UNACAF-70 con $1111.2 \mathrm{~g}$ en promedio por planta y fue estadísticamente diferente a UNACAF-223 con $34.2 \mathrm{~g}$ en promedio por planta que obtuvo el menor valor (Tabla 2). El promedio fue de $330 \mathrm{~g} /$ planta $(35.9 \mathrm{qq} / \mathrm{ha})$ y se tuvo 38 accesiones que produjeron más que este valor. UNACAF-70 y UNACAF-223 corresponderían a las variedades Catimor y Borbón, colectada en Junín y Puno, respectivamente [10]. En otro estudio se encontró en 71 accesiones de café un peso promedio de $450 \mathrm{~g} /$ planta de café pergamino seco [4].

Si analizamos los resultados encontramos que las accesiones más sobresalientes corresponden a las variedades Catimor y Caturra, con una producción de más de $1000 \mathrm{~g} /$ planta de café pergamino seco que equivalen a más de 100 qq/ha, cifra que está por encima del promedio nacional estimado en 14 qq/ha. Sin embargo, estas variedades también son encontradas en accesiones con menores rendimientos. Estos resultados contradictorios se podrían explicar por diversos factores tales como: genético, fertilidad, plagas y enfermedades, que deberían ser estudiados.

\subsection{Peso de 100 semillas}

Los resultados del análisis de variancia indican que hubo diferencias significativas entre los tratamientos, el coeficiente de variabilidad es de $11.98 \%$. UNACAF-187 obtuvo un peso promedio de 23.5 y fue estadísticamente diferente a la accesión UNACAF-197 con $12.5 \mathrm{~g}$ en promedio que obtuvo el menor valor (Tabla 2). La diferencia entre los valores extremos fue de $11 \mathrm{~g}$ y el peso promedio de 100 semillas para las accesiones en el ensayo fue de $15.61 \mathrm{~g}$, existiendo 37 accesiones con un peso mayor a este. UNACAF-187 y UNACAF-197, corresponderían a las variedades Maragogype y NN respectivamente [10] Asimismo en Costa Rica se encontró que el peso de 100 semillas de café para la variedad Caturra oscilaba entre 16 y 18 gramos [8].

\subsection{Relación CC/CPS}

Esta variable representa la cantidad de café cerezo requerido para obtener un kilogramo de café pergamino seco, algunos estudios encontraron para la variedad Catimor valores entre 4.89 y 4.94 en Colombia [14]; mientras que en nuestro país se obtuvo en la variedad Caturra Roja una relación CC/CPS de 5.00 [4]. En este ensayo, UNACAF147 con 5.88 en promedio fue estadísticamente diferente a UNACAF-144 que tuvo una relación CC/CPS de 3.89 y obtuvo el menor valor (Tabla 2). UNACAF-147 y UNACAF-144 corresponderían a las variedades Catimor y Villalobos, respectivamente [10]. El valor promedio de la relación CC/CPS fue 4.94 y hubieron 52 accesiones con un valor mayor a este.

\subsection{Incidencia de roya}

La roya del café (Hemileia vastatrix), es considerada la enfermedad más devastadora del cultivo de café [1]. En este estudio se encontró diferencias significativas entre los tratamientos y el coeficiente de variabilidad fue de $30.30 \%$. La incidencia estuvo entre 0 y $97.27 \%$, pero en 28 accesiones no se reportó la enfermedad (Tabla 2) y corresponderían en general a las variedades Catimor y Gran Colombia [10], conocidas como resistentes a la roya [2].

Esta enfermedad causa que la planta se debilite, las hojas enfermas se caen y la producción de café disminuye considerablemente [6]; sin embargo en este estudio UNACAF-120 presentó el tercer mejor rendimiento a pesar de tener un $87.75 \%$ de incidencia de roya. Se comparó la incidencia de roya con el rendimiento de café y se encontró en la localidad de Villa Rica la mayor incidencia, pero contrariamente a lo esperado también se encontró el más alto rendimiento [11]. El desarrollo de las enfermedades de plantas cultivadas es un fenómeno difícil de explicar [7] ya que intervienen diversos factores como: la lluvia, temperatura, carga fructífera, cosecha de los frutos, inóculo residual, entre otros [11]. 


\subsection{Nivel de infestación de broca}

La broca del café (Hypothenemus hampei), es considerada la plaga más importante en todos los países cafetaleros [5], es por ello que SENASA recomienda un programa de manejo integrado para el control de broca. Un componente fundamental en este tipo de estrategias son las variedades resistentes, sin embargo todas las variedades de Coffea son atacadas por este insecto [16].

En esta evaluación se encontró diferencias significativas entre los tratamientos y el coeficiente de variabilidad fue de $24.05 \%$. El nivel de infestación en promedio estuvo entre 0 y $21.92 \%$ y el promedio fue de $7.98 \%$. La prueba de Duncan a un nivel de 0.05 indica que la accesión con el mayor nivel de infestación fue UNACAF-139 con $21.92 \%$ en promedio y fue estadísticamente diferente a UNACAF-96 que tuvo un nivel de infestación de 1.07\% (Tabla 2).

UNACAF-139 y UNACAF-96 corresponderían a las variedades Catimor y Caturra, respectivamente [10]. Es importante mencionar que todas las accesiones que presentaron un nivel de infestación de broca mayor al 19\% corresponderían sólo a la variedad Catimor (Tabla 2)

\subsection{Análisis de componentes principales}

Los caracteres con mayor contribución sobre los componentes principales $(\geq 0.70)$ y por ende con mayor aporte en la variabilidad de materiales de café han sido siete (peso de café cerezo, peso de café pergamino seco, número de frutos por planta, peso de pulpa de 100 frutos, peso de 100 frutos, nivel de infestación de broca y número de cosechas) (Tabla 3).

Las variables relación CC/CPS, incidencia de roya y peso de 100 semillas fueron los descriptores que explican $0.61,0.55,0.20$ respectivamente de la variancia en los tres primeros componentes, se les puede considerar como los menos discriminantes.

\subsection{Análisis del agrupamiento}

En la Figura 3, el dendrograma obtenido a partir de las distancias consideradas entre 95 accesiones y determinadas mediante 10 caracteres, ha resultado con un coeficiente cofenético de 0.75 . Se diferencia claramente a dos grupos.
La primera agrupación es representada por una sola accesión UNACAF-223, la cual difiere del resto.

El segundo grupo se subdivide en dos grupos, la primera conformada UNACAF-151, UNACAF-187, UNACAF-193, UNACAF-172, UNACAF-200, UNACAF-223 que presentan valores semejantes en caracteres con respecto a la capacidad productiva de la planta; el segundo subgrupo está conformada por el mayor número de accesiones en donde resaltan grupos agronómicos como Típica con bajos rendimientos y alta incidencia de roya y los grupos de Caturra y Catimor con altos rendimientos y diferentes respuesta a la incidencia de roya. Dicho conjunto al contener casi todas la accesiones evaluadas en este estudio, dificulta una clara delimitación entre grupos agronómicos; esto a consecuencia de una alta variabilidad con respecto a los diferentes caracteres estudiados.

\section{Conclusiones}

Teniendo en cuenta las condiciones en las que se efectúo este trabajo, se concluye:

Los factores genéticos y ambientales ocasionaron comportamientos variables en las diferentes accesiones de café. En el análisis de componentes principales, se encontró que las variables peso de café cerezo, peso de café pergamino seco y número de frutos aportaron más a la expresión de la variación de las 95 accesiones.

Existieron 64 accesiones con rendimientos de café pergamino seco considerados bajos (34-398 g/planta), 28 con rendimientos medios (400-718 $\mathrm{g} /$ planta) y 03 accesiones con rendimientos altos (800-1100 g/planta).

Las accesiones con un nivel de incidencia de roya mayor a $90 \%$ corresponden a las variedades Típica y Bourbon. Sin embargo, existieron accesiones que no presentaron síntomas de la enfermedad, como Catimor y Gran Colombia. El nivel de incidencia de roya fue mayor en el nivel inferior, seguido por el nivel medio y finalmente el nivel superior de la planta.

En la última evaluación se encontraron accesiones con $100 \%$ de incidencia de roya correspondientes a las variedades de Típica, Bourbon y Caturra. Estas plantas se encontraron totalmente defoliadas. 
Tabla 2: Resultados de las variables evaluadas en 95 accesiones de café en el Banco de Germoplasma, Chanchamayo 2016.

\begin{tabular}{|c|c|c|c|c|c|c|c|}
\hline $\begin{array}{l}\text { Código de } \\
\text { accesión }\end{array}$ & Variedad & $\begin{array}{l}\text { Peso de CPS } \\
\text { (g/planta) }\end{array}$ & $\begin{array}{c}\text { Peso de } \\
\text { CPS (qq/ha) }\end{array}$ & $\begin{array}{l}\text { Relación } \\
\text { CC/CPS }\end{array}$ & $\begin{array}{l}\text { Peso de } 100 \\
\text { semillas (g) }\end{array}$ & $\begin{array}{c}\text { Nivel de } \\
\text { Incidencia } \\
\text { de Roya (\%) }\end{array}$ & $\begin{array}{c}\text { Nivel de } \\
\text { Infestación } \\
\text { de Broca (\%) }\end{array}$ \\
\hline UNACAF-1 & Caturra & 424.10 & 46.10 & 5.02 & 16.80 & 35.58 & 3.00 \\
\hline UNACAF-3 & Bourbon & 533.66 & 58.01 & 4.79 & 14.10 & 28.01 & 9.08 \\
\hline UNACAF-10 & Catuaí & 400.26 & 43.51 & 5.02 & 15.20 & 55.03 & 14.16 \\
\hline UNACAF-11 & G. Colombia & 301.62 & 32.78 & 4.83 & 14.80 & 0.00 & 12.12 \\
\hline UNACAF-12 & Catimor & 550.46 & 59.83 & 5.36 & 15.20 & 0.00 & 13.60 \\
\hline UNACAF-14 & Caturra & 409.28 & 44.49 & 5.07 & 16.20 & 64.56 & 5.50 \\
\hline UNACAF-15 & Catimor & 203.92 & 22.17 & 5.20 & 14.10 & 0.00 & 21.44 \\
\hline UNACAF-16 & Típica & 274.66 & 29.85 & 4.61 & 16.10 & 70.31 & 4.43 \\
\hline UNACAF-17 & Caturra & 293.38 & 31.89 & 5.03 & 14.80 & 69.96 & 1.47 \\
\hline UNACAF-20 & Pache & 139.22 & 15.13 & 5.44 & 16.60 & 42.82 & 2.30 \\
\hline UNACAF-22 & Catuaí & 108.24 & 11.77 & 5.08 & 13.30 & 50.82 & 5.20 \\
\hline UNACAF-24B & Catuaí & 300.54 & 32.67 & 4.83 & 16.60 & 15.13 & 14.72 \\
\hline UNACAF-28 & Caturra & 283.04 & 30.77 & 5.01 & 15.20 & 12.81 & 10.68 \\
\hline UNACAF-31 & Catimor & 172.96 & 18.80 & 4.93 & 17.20 & 0.00 & 5.53 \\
\hline UNACAF-32 & Catimor & 124.16 & 13.50 & 5.39 & 14.70 & 0.00 & 4.40 \\
\hline UNACAF-35 & Caturra & 398.40 & 43.30 & 5.13 & 15.60 & 20.96 & 2.93 \\
\hline UNACAF-41 & Mundo Novo & 182.76 & 19.87 & 4.74 & 16.20 & 70.26 & 12.52 \\
\hline UNACAF-42 & Pache & 413.54 & 44.95 & 4.66 & 14.60 & 79.44 & 8.37 \\
\hline UNACAF-46 & Caturra & 386.88 & 42.05 & 5.42 & 12.80 & 74.07 & 4.62 \\
\hline UNACAF-51 & Caturra & 671.10 & 72.95 & 5.26 & 13.80 & 4.28 & 16.68 \\
\hline UNACAF-60 & Caturra & 228.54 & 24.84 & 4.81 & 14.20 & 19.46 & 3.27 \\
\hline UNACAF-63 & Catimor & 178.70 & 19.42 & 5.35 & 15.10 & 0.00 & 15.35 \\
\hline UNACAF-67 & Caturra & 75.42 & 8.20 & 5.27 & 16.90 & 61.84 & 16.22 \\
\hline UNACAF-68 & Catimor & 211.68 & 23.01 & 5.25 & 19.20 & 0.00 & 14.90 \\
\hline UNACAF-70 & Catimor & 1111.26 & 120.79 & 4.89 & 13.50 & 0.00 & 19.84 \\
\hline UNACAF-74 & Cavimor & 443.10 & 48.16 & 5.51 & 14.50 & 0.00 & 19.20 \\
\hline UNACAF-76 & Típica & 103.78 & 11.28 & 4.12 & 15.90 & 83.13 & 3.07 \\
\hline UNACAF-79 & Colombia & 183.94 & 19.99 & 5.09 & 14.20 & 0.00 & 7.00 \\
\hline UNACAF-80 & Catimor & 452.60 & 49.20 & 5.00 & 14.60 & 0.00 & 7.45 \\
\hline UNACAF-82 & Caturra & 253.12 & 27.51 & 4.97 & 16.20 & 86.93 & 6.75 \\
\hline UNACAF-83 & Caturra & 239.70 & 26.05 & 4.74 & 13.80 & 82.26 & 1.37 \\
\hline UNACAF-85 & Catimor & 607.30 & 66.01 & 5.02 & 15.30 & 0.00 & 9.35 \\
\hline UNACAF-92 & Caturra & 183.26 & 19.92 & 5.14 & 16.10 & 77.70 & 2.55 \\
\hline UNACAF-95 & Caturra & 219.00 & 23.80 & 4.83 & 17.10 & 85.00 & 4.60 \\
\hline UNACAF-96 & Caturra & 154.84 & 16.83 & 5.07 & 16.60 & 58.63 & 1.07 \\
\hline UNACAF-102 & Caturra & 314.40 & 34.17 & 4.87 & 14.10 & 75.81 & 1.67 \\
\hline UNACAF-105 & Típica & 164.42 & 17.87 & 4.67 & 14.90 & 48.10 & 4.90 \\
\hline UNACAF-107 & Caturra & 488.04 & 53.05 & 4.59 & 15.40 & 19.91 & 2.00 \\
\hline UNACAF-108 & Catimor & 463.18 & 50.35 & 5.26 & 15.20 & 40.40 & 18.08 \\
\hline UNACAF-109 & Catimor & 126.16 & 13.71 & 5.70 & 17.60 & 24.95 & 5.00 \\
\hline UNACAF-114 & Catimor & 373.66 & 40.62 & 5.73 & 15.40 & 0.00 & 20.56 \\
\hline UNACAF-116 & Catimor & 409.92 & 44.56 & 5.67 & 16.00 & 0.00 & 21.16 \\
\hline UNACAF-120 & Caturra & 810.46 & 88.09 & 4.73 & 13.50 & 87.75 & 2.45 \\
\hline UNACAF-123 & Caturra & 480.46 & 52.22 & 4.76 & 14.20 & 74.97 & 1.33 \\
\hline UNACAF-125 & Caturra & 682.12 & 74.14 & 4.73 & 15.50 & 63.49 & 1.53 \\
\hline UNACAF-129 & Bourbon & 327.16 & 35.56 & 4.98 & 13.00 & 49.74 & 6.79 \\
\hline UNACAF-132 & Típica & 105.88 & 11.51 & 4.85 & 14.10 & 93.28 & 4.50 \\
\hline UNACAF-133 & Catimor & 585.08 & 63.60 & 5.35 & 14.90 & 0.00 & 10.40 \\
\hline UNACAF-135 & Catimor & 355.04 & 38.59 & 5.37 & 15.70 & 0.00 & 17.72 \\
\hline UNACAF-137 & Catimor & 632.82 & 68.78 & 5.45 & 16.10 & 0.00 & 16.55 \\
\hline UNACAF-139 & Catimor & 262.56 & 28.54 & 5.65 & 20.10 & 0.00 & 21.92 \\
\hline UNACAF-141 & Caturra & 203.00 & 22.07 & 5.50 & 12.70 & 0.00 & 1.47 \\
\hline UNACAF-143 & Geisha & 208.84 & 22.70 & 3.97 & 18.80 & 97.27 & 3.53 \\
\hline UNACAF-144 & Villalobos & 176.32 & 19.17 & 3.90 & 15.80 & 68.52 & 1.70 \\
\hline UNACAF-147 & Catimor & 556.24 & 60.46 & 5.88 & 14.90 & 0.00 & 4.40 \\
\hline UNACAF-149 & Catimor & 898.94 & 97.71 & 4.64 & 21.80 & 1.01 & 8.10 \\
\hline UNACAF-151 & Típica & 54.38 & 5.91 & 4.20 & 15.10 & 91.29 & 4.20 \\
\hline UNACAF-153 & Bourbon & 162.34 & 17.65 & 4.81 & 18.10 & 0.00 & 3.33 \\
\hline UNACAF-157 & Cape & 287.84 & 31.29 & 5.13 & 15.30 & 58.92 & 2.33 \\
\hline UNACAF-159 & Bourbon & 555.74 & 60.41 & 4.74 & 17.80 & 0.00 & 2.00 \\
\hline UNACAF-165 & Típica & 111.36 & 12.10 & 4.06 & 15.20 & 90.68 & 3.40 \\
\hline UNACAF-166 & Catimor & 718.10 & 78.05 & 4.80 & 15.40 & 0.00 & 5.86 \\
\hline UNACAF-167 & G. Colombia & 301.88 & 32.81 & 4.77 & 14.80 & 7.25 & 6.35 \\
\hline UNACAF-168 & Pache & 346.96 & 37.71 & 4.03 & 16.30 & 86.43 & 13.32 \\
\hline UNACAF-172 & Bourbon & 114.64 & 12.46 & 4.49 & 18.20 & 90.23 & 2.53 \\
\hline
\end{tabular}




\begin{tabular}{|c|c|c|c|c|c|c|c|}
\hline $\begin{array}{l}\text { Código de } \\
\text { accesión }\end{array}$ & Variedad & $\begin{array}{c}\text { Peso de CPS } \\
\text { (g/planta) }\end{array}$ & $\begin{array}{c}\text { Peso de } \\
\text { CPS (qq/ha) }\end{array}$ & $\begin{array}{l}\text { Relación } \\
\text { CC/CPS }\end{array}$ & $\begin{array}{c}\text { Peso de } 100 \\
\text { semillas }(g)\end{array}$ & $\begin{array}{c}\text { Nivel de } \\
\text { Incidencia } \\
\text { de Roya (\%) }\end{array}$ & $\begin{array}{c}\text { Nivel de } \\
\text { Infestación } \\
\text { de Broca (\%) }\end{array}$ \\
\hline UNACAF-173 & CR 95 & 175.56 & 19.08 & 5.01 & 16.20 & 4.18 & 3.67 \\
\hline UNACAF-179 & NN & 332.62 & 36.15 & 4.40 & 13.40 & 49.16 & 3.40 \\
\hline UNACAF-180 & Catimor & 503.46 & 54.72 & 5.23 & 15.30 & 0.00 & 21.36 \\
\hline UNACAF-181 & Caturra & 569.80 & 61.93 & 4.85 & 13.90 & 53.10 & 1.62 \\
\hline UNACAF-182 & Caturra & 220.58 & 23.98 & 4.91 & 15.20 & 6.30 & 3.13 \\
\hline UNACAF-183 & Bourbon & 245.68 & 26.70 & 4.78 & 18.00 & 70.66 & 4.20 \\
\hline UNACAF-185 & Catimor & 464.58 & 50.50 & 5.34 & 15.30 & 43.88 & 19.04 \\
\hline UNACAF-187 & Maragogype & 49.36 & 5.37 & 5.00 & 23.50 & 83.47 & 1.60 \\
\hline UNACAF-188 & Caturra & 255.80 & 27.80 & 4.95 & 14.50 & 23.21 & 3.90 \\
\hline UNACAF-192 & Bourbon & 199.32 & 21.67 & 4.83 & 14.10 & 62.89 & 1.47 \\
\hline UNACAF-193 & NN & 64.22 & 6.98 & 4.62 & 13.80 & 85.70 & 1.50 \\
\hline UNACAF-196 & Caturra & 517.66 & 56.27 & 5.07 & 17.20 & 67.40 & 12.24 \\
\hline UNACAF-197 & NN & 124.98 & 13.58 & 4.46 & 12.50 & 73.78 & 2.40 \\
\hline UNACAF-200 & NN & 64.62 & 7.02 & 4.25 & 14.40 & 4.82 & 1.60 \\
\hline UNACAF-202 & Brasilero & 224.08 & 24.36 & 5.61 & 18.50 & 8.90 & 14.40 \\
\hline UNACAF-203 & NN & 247.18 & 26.87 & 4.94 & 14.40 & 45.63 & 18.28 \\
\hline UNACAF-207 & Caturra & 648.56 & 70.50 & 5.20 & 13.30 & 72.67 & 11.96 \\
\hline UNACAF-208 & Caturra & 397.24 & 43.18 & 5.06 & 13.80 & 69.85 & 2.40 \\
\hline UNACAF-210 & Catimor & 289.18 & 31.43 & 5.52 & 15.10 & 0.00 & 21.36 \\
\hline UNACAF-211 & Caturra & 407.28 & 44.27 & 5.16 & 15.80 & 71.19 & 2.65 \\
\hline UNACAF-213 & Geisha & 345.34 & 37.54 & 4.28 & 19.50 & 0.00 & 4.47 \\
\hline UNACAF-215 & Catimor & 279.58 & 30.39 & 5.24 & 16.50 & 0.00 & 18.44 \\
\hline UNACAF-216 & Típica & 310.12 & 33.71 & 4.42 & 15.50 & 85.83 & 4.33 \\
\hline UNACAF-219 & Caturra & 166.80 & 18.13 & 5.12 & 15.10 & 32.40 & 11.57 \\
\hline UNACAF-220 & Catimor & 486.00 & 52.83 & 5.19 & 17.40 & 0.00 & 21.12 \\
\hline UNACAF-222 & Villalobos & 280.20 & 30.46 & 4.59 & 16.10 & 83.58 & 2.00 \\
\hline UNACAF-223 & Bourbon & 34.18 & 3.72 & 4.31 & 18.30 & 91.19 & 2.90 \\
\hline UNACAF-226 & Típica & 174.58 & 18.98 & 4.57 & 14.40 & 94.60 & 2.50 \\
\hline UNACAF-228 & Típica & 224.40 & 24.39 & 4.48 & 14.60 & 82.60 & 3.20 \\
\hline UNACAF-230 & Caturra & 610.84 & 66.40 & 4.95 & 14.60 & 76.77 & 11.68 \\
\hline
\end{tabular}
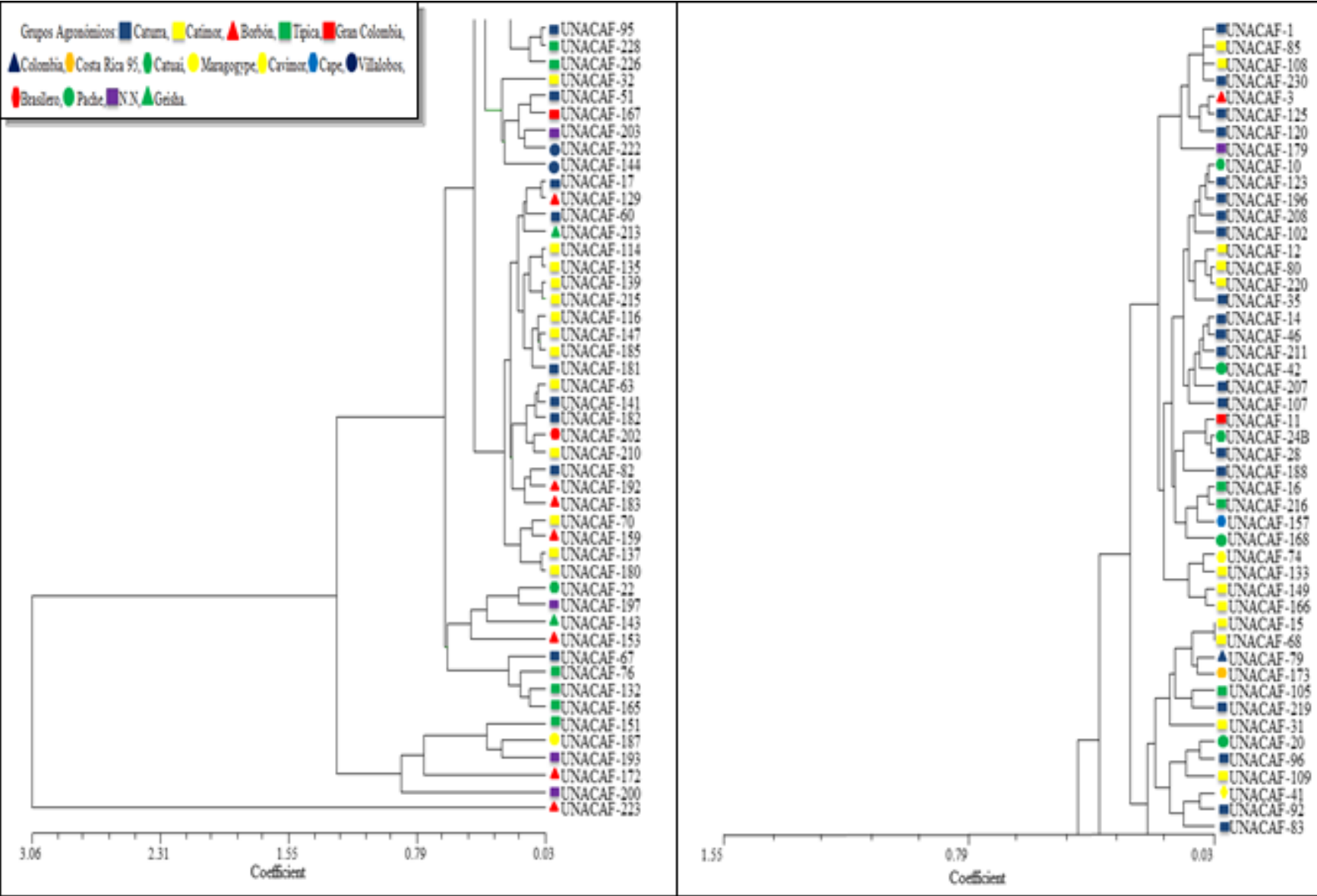

Figura 3: Dendrograma de las 95 accesiones de café evaluadas en la campaña cafetalera 2015-2016 
Tabla 3: Proporción de la varianza explicada por cada descriptor sobre los tres primeros componentes principales.

\begin{tabular}{|c|l|c|}
\hline № & \multicolumn{1}{|c|}{$\begin{array}{c}\text { Descriptor } \\
\text { Agronómico }\end{array}$} & $\begin{array}{c}\text { Proporción } \\
\text { de la } \\
\text { Variancia }\end{array}$ \\
\hline 1 & $\begin{array}{l}\text { Peso de café cerezo } \\
\text { (g) }\end{array}$ & 0.98903 \\
\hline 2 & $\begin{array}{l}\text { Peso de café } \\
\text { pergamino seco (g) }\end{array}$ & 0.98723 \\
\hline 3 & $\begin{array}{l}\text { Número de frutos por } \\
\text { planta }\end{array}$ & 0.97402 \\
\hline 4 & $\begin{array}{l}\text { Peso de pulpa de 100 } \\
\text { frutos maduros (g) }\end{array}$ & 0.96758 \\
\hline 5 & $\begin{array}{l}\text { Peso de 100 frutos } \\
\text { maduros (g) }\end{array}$ & 0.96758 \\
\hline 6 & $\begin{array}{l}\text { Nivel de infestación de } \\
\text { Broca (\%) }\end{array}$ & 0.76976 \\
\hline 7 & Número de cosechas & 0.76290 \\
\hline 8 & Relación CC/CPS & 0.61903 \\
\hline 9 & $\begin{array}{l}\text { Incidencia de Roya } \\
\text { (\%) }\end{array}$ & 0.55124 \\
\hline 10 & Peso de 100 semillas & 0.20777 \\
\hline
\end{tabular}

Nueves accesiones presentaron un nivel de infestación de broca mayor a 19\%, todas correspondieron a la variedad Catimor.

\section{Referencias}

[1] G. Agrios, Plant Pathology, 4th ed., (Academic Press, California, 1997).

[2] ANACAFE (Asociación Nacional del Café), Manual de la caficultura, $3^{\circ} \mathrm{Ed}$. Guatemala, (1998).

[3] F. Anthony, M. Combes, C. Astorga, B. Bertrand, G. Graziosi, P. Lashermes, Theor. Appl. Genet., 104 (2002) 894-900
[4] A. Apaza, Ing. Agr., Tesis, Universidad Nacional Agraria La Molina, Lima, Perú, 2013.

[5] S. Fernández, J. Cordero, Bioagro 17 (2007) 143-148.

[6] FONAIAP (Fondo Nacional de Investigadores Agropecuarios), Roya del cafeto, Venezuela, (1984).

[7] A. Gárate y I. Bonilla, en Fundamentos de Fisiología Vegetal, editado por Azcón-Bieto y Talón, $2^{\circ}$ Ed. (Universitat de Barcelona. Madrid, 2001).

[8] J. Herrera, R. Alizaga, G. Alizaga, Agro. Cost., 17(1) (1993) 25-32.

[9] INEI (Instituto Nacional de Estadística e Informática), Resultados Definitivos, IV Censo Nacional Agropecuario (Ministerio de Agricultura y Riego, Lima, Perú, 2012).

[10] A. Julca, R. Blas, R. Borjas, S. Bello, J. Anahui, D. Talaverano, R. Crespo, G. Fundes, Informe de colecta de germoplasma de café en el Perú, (FDA, UNALM, Lima, Perú, 2010).

[11] A. Julca, C. Echevarría, Y. Ladera, R. Borjas, Una revisión sobre la roya del café (Hemileia vastatrix) algunas experiencias $y$ recomendaciones para el Perú, (UNALM, IRD-Selva, Lima, Perú, 2013).

[12] Junta Nacional del Café, El Cafetalero, $N^{\circ}$ 56, Perú, (2016) 41 pp.

[13] L. Marín, P. Arcila, R. Montoya, T. Oliveros, Cenicafé, 54(4) (2003) 297-315

[14] J. Montilla, J. Arcila, M. Aristizábal, E. Montoya, G. Puerta, C. Oliveros, Cenicafé, 59(2) (2008) 120-142

[15] G. Puerta, Estudios de calidad del café de Colombia, según la altitud y los suelos del cultivo, (Cenicafé, Colombia, 2007) 87p.

[16] J. Romero y G. Cortina, Cenicafé, 55(4) (2004) 341-346 